vor. - Das Absetzen und Auswaschen des Niederschlags geschieht in Folge dessen sehr langs a m. Dies veranlasste Fieinus in Bensheim (s. Archiv 1878, Bd. XII, 29), die Fällung durch Eingiessen der Lösung in das dreifache Volumen $90 \%$ gen Weingeistes vorzunehmen. Man erhält so einen Niederschlag von harzähnlicher Beschaffenheit, der die unangenehme Eigenschaft besitzt, sich sehr schwer vollständig von seinem Kochsalzgehalt, welcher bei längerem Stehenlassen den Niederschlag sogar theilweise als Salzkruste bedeckt, befreien zu lassen.

Um diese Klippen zu umgehen, verdünne ich die Lösung mit 83 Gewichtstheilen destillirten Wassers und trage dieselbe dann in ein Gemisch aus je 83 Gewichtstheilen Spiritus und Aq. destillata unter Umrühren ein. Man erhält dann einen fein vertheilten, sich rasch absetzenden Niederschlag, der sich gut und rasch mit dem obigen Weingeistgemisch, zuletzt mit wenig Aq. destillat. auf einem Filter auswaschen lässt, und einen hellbraunen Syrupus ferri oxydati giebt, der vollkommen rein und angenehm schmeckt.

\title{
Ueber das Vorkommen von Krystallen in den Secreten einiger Rhusarten.
}

Von Arthur Meyer in Strassburg.

Burseraceen und Anacardiaceen zeichnen sich bekanntermaassen durch das allgemeine Vorkommen protogener intercellularer Secretcanäle aus. Diese Canäle findet man schon im Embryo mit Secret gefültt, Wurzeln und Cotyledonen als zusammenhängendes System durchziehend. In der erwachsenen Pflanze begleiten sie die Siebröhrenpartien der Gefässbündel bis in die äussersten Bündelenden.

Der Inhalt der Canäle ist bei den verschiedenen Arten dieser Familien sehr verschieden; ich erinnere nur an die officinellen Harze und Gummiharze, welche diesen Secretcanälen ihre Entstehung verdanken, an Myrrha, Olibanum, Mastix und Elemi: Innerhalb enger Verwandtschaftskreise scheint allerdings eine gewisse Uebereinstimmung unter den Secreten zu herrschen. So ist, wenn man nach den Resultaten urtheilen darf, die ich bei Untersuchung einer kleinen Anzahl von Species erhalten habe, das Auftreten von 
A. Mejer, Vorkomm. T. Krystallen i. d. Secreten einiger Rhusarten. 113

Krystallen in dem Secrete bei den Arten der Gattung Rhus ein sehr verbreitetes.

Diese Krystalle, welche in ziemlicher Anzahl, bis zur Grösse von $0,04 \mathrm{~mm}$. und frei in dem Secrete sohwimmend vorkommen, zeigen für die bestimmte Species stets eine bestimmte Form der Ausbildung. Alle, welche ich gefunden, geben dabei folgende Reactionen, durch welche der Beweis geliefert ist, dass in diesen Fällen kein oxalsaurer Kalk vorliegt.

Die Krystalle sind vollständig verbrennlich; sie lösen sich ohne Trübung oder Bildung von neuen Krystallen in Ammoniak, Schwefelsäure, Essigsäure, Kalkwasser, Oxalsäurelösung, dagegen nicht in Weingeist, Chloroform, Wasser; ausserdem sind sie doppeltbrechend, und ihre Schwingungsrichtungen liegen parallel und senkrecht zu den Krystallkanten orientirt.

Die Krystalle der drei sich systematisch nahe stehenden Species, Rhus toxicodendron Mich., vernicifera DC. und succedanea L. (wahrscheinlich wird auch Rhus venenata DC., welche ich nicht untersuchen konnte, dieselben Krystalle enthalten) haben gewöhnlich die Gestalt der Figur I, wobei $a$ die Ansicht der breiten Fläche, $b$ die Kantenansicht darstellt.

Auch zwischen den Secreten der drei Species konnte ich bei mikrochemischer Prüfung keinen Unterschied feststellen. Alle drei sind fast völlig homogen, stark lichtbrechend, lösen sich in Alkohol, Chloroform, Aether fast vollständig, trocknen an der Luft unter Braunfärbung zur festen Masse ein, schwärFig. I.

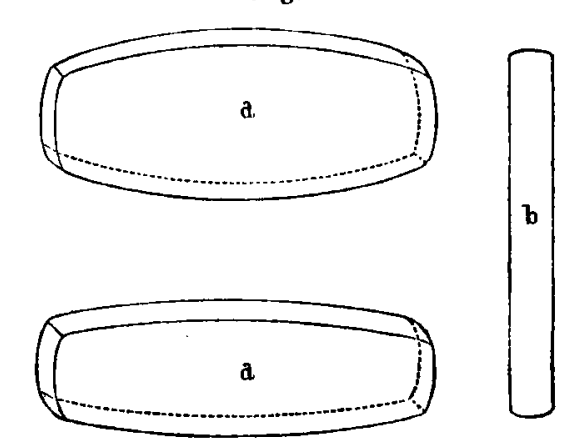
zen sich durch Eisenchlorid intensiv und reduciren Phosphormolybdänsäure, indem sie sich damit tief dunkelblau färben.

Die beiden letzten Reactionen können zur Bestätigung einer Ansicht über den Ort der Entstehung der besprochenen Secrete benutzt werden, und es sei mir gestattet, darüber einige Worte einzuschalten.

Bekanntlich sind die in Rede stehenden Canäle mit einer zartwandigen Zellschicht, dem Epithel, ausgekleidet, von welchem

Arch. d. Pharm. XVII. Bds. 2. Hft. 
man annehmen könnte, dass es das Secret innerhalb des Zellinhaltes erzeuge und durch die Zellmembran in den Intercellularraum hinauspresse. Hier kann man sich nun leicht überzeugen, dass die Epithelzellen in ihrem Inneren keine Spur von Secret enthalten, da sich das kleinste Tröpfchen desselben beim längeren Liegen in sehr verdünnter Eisenchloridlösung durch seine Schwärzung verrathen würde. Es muss also in diesem Falle das Secret wie die Secrete der Drüsenhaare innerhalb der Cellulosemembranen der Epitheizellen gebildet werden. ${ }^{1}$

Die Secrete von Rhus toxicodendron, vernicifera und succedanea haben bekanntermaassen auch die Giftigkeit gemein, und da die Wirkung des Secretes von Rhus vernicifera, dem japanischen Lacke, der Wirkung des Secretes ron Rhus toxicodendron sehr ähnlich ist, bedingen vielleicht dieselben chemischen Substanzen die Giftigkeit dieser verschiedenen Säfte.

Nach der Form der Krystalle schliesst sich zuerst der der Gattung Rhus so nahe stehende Schinus molle L. ${ }^{2}$ an, dann Rhus pubescens Thunb. und Rhus villosa $L$.

Wie die Abbildung dieser Krystalle, Figur II, zeigt, bilden sich hier die Flächen besser aus; doch kommen auch unvollkommenere Fig. II.

Krystalle vor. $b$ stellt die Ansicht des aufrecht stehenden, in der Richtung des Pfeiles betrachteten Krystalles dar.

Das homogene, stark lichtbrechende, schwach aromatisch riechende Secret der beiden letzten Pflanzen verhält sich gegen Eisenchlorid indifferent und löst sich fast völlig in Chloroform.

Auch das Secret von Rhus cotinus L. ist eine klare, gelbliche Flüssigkeit, welche an der Luft etwas dunkler wird, doch sich nicht bräunt, von citronenähnlichem Geruche, leicht löslich in Chloroform, schwer löslich in Terpenthinöl.

1) Siehe hierzu „Vergleichende Anatomie der Vegetationsorgane der Phanerogamen und Farne von A. de Bary. Leipzig 1877, pag. 213 u. 99."

2) Die Drehung, welche Blattstïckchen desselben ausführen, wenn man sie auf Wasser wirft, rührt von dem ausfliessenden und sich wio Oel auf dem Wasser ausbreitenden Secrete her. Uebrigens bewegen sich Blattstüekchen verschiedener Rhusarten ebenfalls mehr oder weniger unter denselben Verhältnissen. 
A. Meyer, Vorkomm. v. Krystallen i. d. Secreten einiger Rhusarten. 115

Die in demselben suspendirten Krystalle zeichnen sich jedoch vor den bis jetzt beschriebenen durch grössere Breite aus. Figur III. zeigt in $a$ den Krystall von der breiten Fläche, in $b$ denselben von oben.

Eine ähnliche Form besitzen die Krystalle von Rhus typhina L., während das Secret derselben sehr verschieden von allen bis jetet betrachteten ist. Es stellt eine feinkörnige, weisse Emulsion dar, welche sich mit Wasser verdünnen lässt. Das bräunliche Harz derselben lässt sich Fig. III.

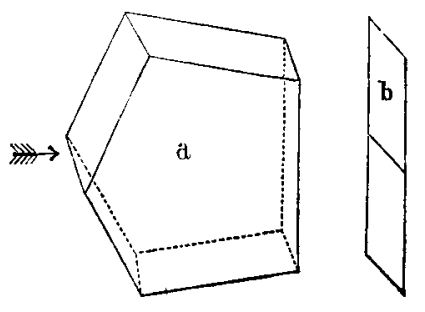
durch Chloroform ausziehen, während in Wasser lösliches Gummi zurïckbleibt. Mit Eisenchlorid schwärzt sich der Milchsaft nicht. Auch das harzige, homogene Secret von Rhus semialata Murr., der Stammpflanze der chinesischen Gallen, ist gerbsäurefrei und zeichnet sich vor allen Secreten der bis jetzt besprochenen Rhusarten durch das Fehlen der Krystalle aus. - Alle erwähnten Krystalle fand ich im August, während des Winters und auch in den Secretgängen der Frühjahrssprossen.

Was andere Terebinthaceen anbelangt, so habe ich nur das Secret von Pistacia Lentiscus L. im frischen Zustande untersucht und darin keine Krystalle gefunden. Auch scheint es, dass die Stammpflanzen des Weihrauchs und der Myrrha keine Krystalle in ihren Secreten enthalten; wenigstens konnte ich niemals in Gummiharzen Krystalle entdecken.

Leider stand mir kein Material von Anacardium occidentale L. und Semecarpus Anacardium L. fil. zur Verfügung. Das dunkle Secret, welches in den Fruchtschalen von Semecarpus Anacardium enthalten ist, zeigte allerdings kugelige Aggregate nadelförmiger Krystalle, doch ist damit durchaus nicht gesagt, dass in dem Secrete der lebenden Pflanze solche vorkommen. Eine Probe des Secretes von Melanorrhoea usitata Wall. (ich verdanke sie der Güte des Herrn Professor Flückiger), welches wie das Secret von Rhus vernicifera als Lack gebraucht wird, ${ }^{1}$ zeigte keine Krystalle.

1) Wallich, Plantae asiaticae rariores, 1830. Vol. I, pag. 9. 